\section{Prevalência estimada e fatores associados à hipertensão arterial em indígenas adultos Krenak do Estado de Minas Gerais, Brasil}

\author{
Estimated prevalence of hypertension and \\ associated factors in Krenak indigenous \\ adults in the state of Minas Gerais, Brazil
}

Prevalencia estimada y factores asociados a la hipertensión arterial en indígenas adultos Krenak del Estado de Minas Gerais, Brasil

\author{
Cristiane Alvarenga Chagas 1 \\ Teresa Gontijo de Castro 2 \\ Maurício Soares Leite 3 \\ Maria Augusta Corrêa Barroso Magno Viana 1 \\ Mark Anthony Beinner 1 \\ Adriano Marçal Pimenta 1
}

\section{Resumo}

Estudo transversal realizado em 2016, cujo objetivo foi descrever a prevalência estimada e os fatores associados à hipertensão arterial sistêmica entre adultos e idosos Krenak, em Terra Indígena localizada na beira do rio Doce, na região leste de Minas Gerais, Brasil. Foram aferidos peso, estatura, perímetro da cintura, pressão arterial sistólica, pressão arterial diastólica e glicemia capilar. Informações sociodemográficas e de estilo de vida foram obtidas via aplicação de questionário face a face. Modelos de regressão de Poisson foram construídos para estimar a associação independente entre as variáveis de interesse e a hipertensão arterial sistêmica. A prevalência da hipertensão arterial sistêmica foi de 31,2\% (IC95\%: 24,4-37,9) entre os indígenas Krenak. Houve associação independente no modelo final para o aumento da idade, obesidade abdominal e hiperglicemia. Destaca-se a necessidade de ações eficazes de prevenção, de diagnóstico e acompanhamento frente aos fatores modificáveis da hipertensão arterial sistêmica, uma vez que foi observada elevada prevalência dessa condição na comunidade indígena Krenak.

Hipertensão; Índios Sul-americanos; Fatores de Risco; Avaliação Nutricional; Enfermagem em Saúde Comunitária

\author{
Correspondência \\ C. A. Chagas \\ Escola de Enfermagem, Universidade Federal de Minas Gerais. \\ Av. Alfredo Balena 190, 4o andar, sala 422, Belo Horizonte, MG \\ 30130-100, Brasil. \\ cristianebyt@hotmail.com \\ ${ }^{1}$ Escola de Enfermagem, Universidade Federal de Minas Gerais, \\ Belo Horizonte, Brasil. \\ 2 School of Population Health, The University of Auckland, \\ Auckland, New Zealand. \\ 3 Departamento de Nutrição, Universidade Federal de Santa \\ Catarina, Florianópolis, Brasil.
}




\section{Introdução}

Os indígenas representam mais de 5 mil culturas diversas distribuídas em 90 países, e globalmente há um grande distanciamento dos indicadores sociais e de saúde quando comparadas às populações não indígenas 1,2. Apesar de corresponderem a somente $5 \%$ da população global, $15 \%$ da pobreza mundial se concentra no segmento indígena, o que impacta nas disparidades sociais e de saúde e contribui com a alta carga de doenças crônicas não transmissíveis (DCNT), verificada entre os diferentes povos 1,3. Apesar de o Brasil ter uma pequena porcentagem de indígenas $(0,4 \%$ da população total) quando comparada a outros países latino-americanos 4, existe uma grande heterogeneidade étnica, cultural, linguística, socioeconômica e de localização das Terras Indígenas (TI), refletindo em distintos hábitos alimentares e de vida 5 .

A hipertensão arterial sistêmica (HAS), presente no conjunto das DCNT, apresenta proporções epidêmicas em todo o mundo, sendo responsável pelo aumento da carga de doenças e perda da expectativa de vida devido às incapacidades geradas em decorrência dessa condição crônica ${ }^{6}$. No Brasil, a HAS acomete de $24,3 \%$ a $32,5 \%$ dos adultos 7,8 . De modo similar, a HAS também tem sido encontrada entre os indígenas brasileiros. Apesar de os primeiros estudos conduzidos com adultos indígenas brasileiros terem verificado ausência e/ou níveis pressóricos inferiores aos da população não indígena 9,10,11,12, o quadro atual da epidemiologia da HAS entre os indígenas tem se modificado. O 1o Inquérito Nacional de Saúde e Nutrição dos Povos Indígenas (INSNPI), realizado entre 2008-2009, constatou prevalência de 13,2\% de HAS entre mulheres não gestantes (14 a 49,9 anos de idade), com valores oscilantes de 3,6\% na Região Norte e 17,5\% na Centro-oeste 5 . Estudos pontuais mais recentes 13,14,15 apontaram valores pressóricos elevados entre adultos indígenas, com maiores magnitudes que o observado para não indígenas. As prevalências reportadas de HAS oscilaram de 15,4\% entre a etnia Nahukwá 16 a 46,2\% na etnia Kaingang 14.

A emergência e a magnitude das DCNT entre os povos indígenas são consequências de um cenário epidemiológico complexo de mudanças significativas nos modos de vida entre as sociedades indígenas e que incluem: a expansão de fronteiras agrárias, a degradação ambiental, a restrição dos territórios tradicionalmente percorridos, os conflitos pela terra e o crescente contato com não indígenas. Ainda que com nuances locais, somam-se a tal quadro a proximidade dos centros urbanos e o acesso facilitado a alimentos ultraprocessados, as modificações na maneira de trabalhar e a redução da atividade física 17,18 .

Os estudos que abordam HAS entre os povos indígenas ainda são incipientes, e os já realizados se concentram em sua maior parte nas regiões Norte e Centro-oeste do país ${ }^{13}$. No Estado de Minas Gerais, onde há 13 etnias indígenas pertencentes ao tronco linguístico Macro-Jê, com um contingente populacional de aproximadamente 13 mil pessoas 19,20, até a presente data não há registro de estudos que avaliaram a prevalência de HAS e seus determinantes entre os indígenas. Portanto, este estudo teve por objetivo descrever a prevalência estimada e os fatores associados à HAS entre adultos e idosos Krenak, em TI localizada na beira do Rio Doce, na região leste de Minas Gerais. O estudo pretende contribuir na descrição do cenário da epidemiologia da HAS entre os povos indígenas brasileiros, de forma a subsidiar ações governamentais que visem à redução da ocorrência de DCNT que vem aumentando nesse segmento de forma acelerada.

\section{Metodologia}

Trata-se de um estudo transversal com a população Krenak com 18 anos ou mais de idade de todas as cinco aldeias, da TI Krenak, localizada no Município de Resplendor, vale do Rio Doce, Minas Gerais.

\section{População de estudo}

Os Krenak ou Borún pertencem ao tronco linguístico Macro-Jê, são falantes da língua Borum e constituem os últimos Botocudos do Leste, nome conferido pelos portugueses do século XVIII aos grupos que usavam botoques auriculares e labiais 20 . À época do primeiro contato, eram predominantemente caçadores e coletores seminômades, com organização social caracterizada pelo constante fraciona- 
mento do grupo, pela divisão do trabalho por sexo e idade e um sistema religioso centrado na figura dos Marét e dos Nanitiong. Tiveram como território original a Mata Atlântica no Baixo Recôncavo Baiano, sendo expulsos da área litorânea pelos Tupi 20.

Os primeiros contatos dos Krenak com os colonizadores se deram à época do declínio do ciclo do ouro (século XVI) quando houve deslocamento dos Krenak em direção ao sul, na região do Rio Doce, entre os estados de Minas Gerais e Espírito Santo. Desde então, foram acusados de serem agressivos, insubmissos, e antropofágicos, sendo essas acusações utilizadas para a justificativa de muitas guerras entre os colonizados e os Krenak 20. Ao longo das décadas, os Krenak sofreram com constantes brigas territoriais, sendo quase dizimados. Somente no ano de 1997, houve a restituição de suas terras, com a demarcação de quatro mil hectares no Município de Resplendor, conforme decisão judicial do Supremo Tribunal Federal, que solicitou a anulação dos títulos emitidos ilegalmente pelo governo mineiro 20 .

$\mathrm{Na}$ data presente, o grande desafio enfrentado pelos Krenak diz respeito aos graves prejuízos ambientais causados no Rio Doce pela empresa Samarco S/A, no ano de 2015 (data anterior à coleta de dados desta pesquisa). O acidente provocou a contaminação do Rio Doce com rejeitos de mineração que vazou de uma das barragens da companhia mineradora. O rio era utilizado pelos Krenak para a agricultura, a pesca, a prática de rituais e o lazer. Aproximadamente 126 famílias Krenak que viviam às margens do rio foram afetadas 21.

De acordo com o Censo fornecido pelo Distrito Sanitário Especial Indígena (DSEI) Minas Gerais e Espírito Santo em 2016, o total da população residente em TI Krenak distribuídos nas cinco aldeias com 18 anos ou mais de idade somava 227 indivíduos. Foram convidados a participar do estudo todos os indivíduos com idade $>18$ anos.

\section{Coleta de dados}

A coleta de dados foi realizada em pontos estratégicos existentes nas cinco aldeias como escolas e centros culturais e ocorreu em dois momentos no ano de 2016 (agosto e outubro). As informações foram coletadas nesses locais de forma individual por um grupo de cinco profissionais da saúde treinados (nutricionistas e enfermeiros). Além desses, houve três membros da equipe de saúde indígena local (enfermeiro, dentista e agente de saúde indígena) que auxiliaram na localização e convite para participação no estudo. Os dados foram coletados mediante exame físico (antropometria e aferição da pressão arterial), bioquímico (glicemia capilar) e de questionários (variáveis sociodemográficas, de estilo de vida e hábitos alimentares).

A pressão arterial sistólica (PAS) e a pressão arterial diastólica (PAD) foram obtidas pelo método oscilométrico com utilização de um tensiômetro digital de braço com monitor automático (Omron HEM-7200), e manguitos apropriados ao perímetro do braço do indivíduo. Foram realizadas três aferições no braço direito, com dois minutos de intervalo entre elas, seguindo as recomendações da Sociedade Brasileira de Cardiologia (SBC) 8. Para a análise dos dados, a medida definitiva corresponde à média das duas últimas leituras, sendo a primeira leitura foi desconsiderada. Considerou-se como critério diagnóstico para HAS o ponto de corte definido pela VII Diretriz Brasileira de Hipertensão Arterial 8 que corresponde aos valores de PAS $>140 \mathrm{mmHg}$ e/ou PAD $>90 \mathrm{mmHg}$. Os participantes foram ainda questionados acerca do uso de medicamento para HAS prescrito por médico. Os indivíduos que afirmaram fazer uso deste tipo de medicação foram automaticamente considerados com HAS.

A aferição do peso corporal foi feita por meio de uma balança digital portátil devidamente calibrada (Marte Científica; capacidade 150kg e precisão de 50g). A medida de estatura foi feita em triplicata e obtida por um estadiômetro portátil (Alturaexata; extensão de $2 \mathrm{~m}$ e precisão de $1 \mathrm{~mm}$ ). O perímetro da cintura (PC) também foi medido em triplicata com o auxílio de uma fita métrica inelástica (Sanny; com extensão de $1,50 \mathrm{~m}$ e precisão de $1 \mathrm{~mm}$ ). Valores finais de peso, estatura e PC foram obtidos pela média dos dois valores mais próximos.

A classificação do estado nutricional foi definida segundo o índice de massa corporal (IMC), classificado de acordo com os critérios estabelecidos pela Organização Mundial da Saúde (OMS) 22 para adultos até 59 anos de idade, porém, para o presente estudo, foram dicotomizados em $<24,9 \mathrm{~kg} / \mathrm{m}^{2}$ (sem excesso de peso) e em $>25 \mathrm{~kg} / \mathrm{m}^{2}$ (com excesso de peso); para os indivíduos com 60 anos ou mais, utilizaram-se os critérios propostos por Lipschitz 23 dicotomizados em $<26,9 \mathrm{~kg} / \mathrm{m}^{2}$ (sem excesso de 
peso) e em $>27 \mathrm{~kg} / \mathrm{m}^{2}$ (com excesso de peso). Os valores de $\mathrm{PC}>80 \mathrm{~cm}$ para o sexo feminino e $>94 \mathrm{~cm}$ para o sexo masculino foram classificados como obesidade central 24.

A glicemia casual foi coletada por meio de glicosímetro portátil (Roche Accu-Chek Active), com auxílio de lancetador, lancetas descartáveis e tiras de leituras de glicemia, ambas de uso individual. Os indivíduos foram classificados com glicemia normal quando os valores foram $<99 \mathrm{mg} / \mathrm{dL} \mathrm{em}$ jejum ou $<139 \mathrm{mg} / \mathrm{dL}$ sem jejum e hiperglicemia para valores $>100 \mathrm{mg} / \mathrm{dL}$ em jejum ou $>140 \mathrm{mg} / \mathrm{dL}$ sem jejum 25.

As variáveis demográficas analisadas foram sexo, idade, situação conjugal, enquanto a socioeconômica foi a escolaridade. Para avaliação dos aspectos comportamentais (estilo de vida e hábitos alimentares), foram utilizadas questões-chave que fazem parte do sistema nacional de monitoramento para doenças crônicas conhecido como VIGITEL (Vigilância de Fatores de Risco e Proteção para Doenças Crônicas por Inquérito Telefônico) ${ }^{7}$. No que diz respeito ao estilo de vida, as variáveis analisadas foram o tabagismo, consumo pesado episódico de bebida alcóolica nos últimos 30 dias antes da entrevista, a atividade física realizada em tempo livre e o tempo de tela (total de horas diárias em que o indivíduo passava assistindo à televisão, estava ao computador e/ou ao celular). Quanto aos hábitos alimentares foram analisadas o consumo regular de frutas, hortaliças e feijão, o consumo de refrigerante ou suco artificial, o consumo de carnes com excesso de gordura e a adição de sal à comida pronta.

\section{Análise dos dados}

Em relação à organização das informações, a consolidação de dados foi feita no programa Epi Info, versão 7.1 (https://www.cdc.gov/epiinfo/index.html), por dupla digitação e a análise de consistência no programa Epi Info, versão 3.5.

As análises estatísticas foram realizadas no programa Stata, versão 14 (https://www.stata.com). A caracterização da amostra foi feita com uma análise descritiva das variáveis independentes. Análises bivariadas, utilizando o teste qui-quadrado de Pearson, foram conduzidas de forma a verificar as associações entre a variável dependente HAS, categorizadas de forma dicotômica (sim/não) e as variáveis independentes. As razões de prevalência e seus intervalos de 95\% de confiança (IC95\%) também foram estimados pela regressão de Poisson com variâncias robustas.

Por fim, foram construídos modelos multivariados com a regressão de Poisson com variâncias robustas conforme modelo hierarquizado que seguiu os pressupostos teóricos propostos por Victora et al. 26 e foi previamente testado em estudo sobre fatores associados à HAS em população indígena 27. Em tal modelo (Figura 1), há três blocos de variáveis (bloco 1 - demográficas; bloco 2 - socioeconômicas e comportamentais; bloco 3 - morbidades). Assim, inicialmente, foram introduzidas, no modelo, as variáveis demográficas que apresentaram nível de significância com $\mathrm{p}<0,20$ na análise bivariada, tendo permanecido no modelo aquelas com valor de $\mathrm{p}<0,05$. Na segunda etapa, foram incluídas as variáveis socioeconômicas e comportamentais, utilizando os mesmos valores de significância estatística do primeiro passo. Na terceira etapa, foram acrescidas as variáveis de morbidade, adotando o mesmo critério das etapas anteriores.

\section{Aspectos éticos}

O estudo foi aprovado nas seguintes instâncias: Comitê de Ética e Pesquisa em Seres Humanos da Universidade Federal de Minas Gerais (parecer no 25406413.9.0000.5149), Comissão Nacional de Ética em Pesquisa (CONEP - parecer no 867.977), Conselho Nacional de Desenvolvimento Científico e Tecnológico (CNPq) e Fundação Nacional do Índio (processo no 08620.078.625/2015-86). Líderes indígenas locais foram contatados e forneceram consentimento para o estudo. Todos os indivíduos que participaram da pesquisa assinaram o Termo de Consentimento Livre e Esclarecido (TCLE). Para os que eram analfabetos, foram usadas impressões digitais para documentar a ciência em participação do estudo. Todos os índios que apresentaram alguma alteração significativa no momento da coleta de dados foram encaminhados ao serviço de saúde indígena local. 


\section{Figura 1}

Modelo teórico hierarquizado do estudo dos fatores associados à hipertensão arterial em indígenas Krenak. Resplendor, Minas Gerais, Brasil, 2016.

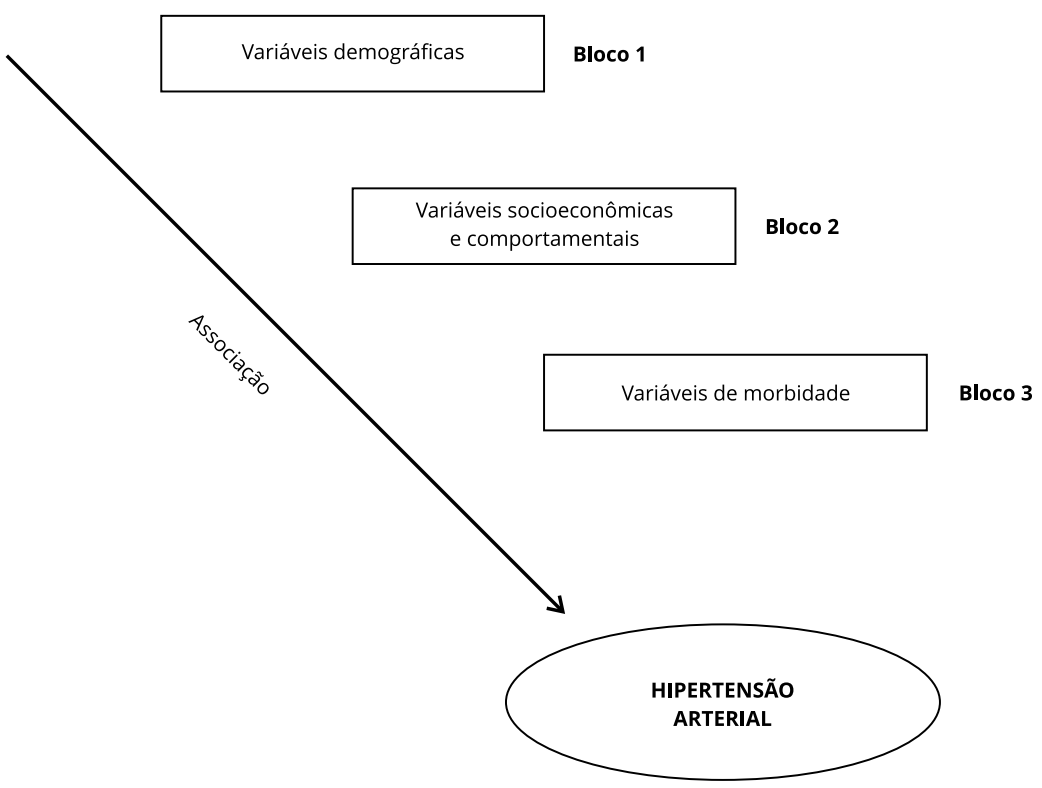

Fonte: elaborado pelos autores.

\section{Resultados}

Dos 227 indígenas convidados a participar do estudo (114 homens e 113 mulheres), houve recusa de 16 pessoas, 18 não foram localizados para aferição dos dados após três tentativas sem sucesso. Além disso, existiram exclusões por incapacidade mental $(n=3)$, mulheres em período gestacional $(n=2)$ e as que não tinham completado um ano de pós-parto $(n=5)$. Portanto, a população final do estudo foi de 183 indígenas adultos e idosos Krenak (77\% do total da população alvo das aldeias).

A prevalência estimada da HAS entre os Krenak foi de 31,2\% (34,4\% para os homens e 27,6\% para as mulheres). Do total de indivíduos entrevistados, $16,5 \%$ informaram que já faziam uso de medicamento anti-hipertensivo (dado não apresentado em tabela).

No que diz respeito às características demográficas e socioeconômicas, a maior parte dos indivíduos que participou da pesquisa era do sexo feminino, estava na faixa etária entre 30 a 39 anos de idade, era casado ou estava em união estável e apresentava mais de 10 anos de estudo. Em relação ao estilo de vida, 14,2\% eram fumantes; $27,3 \%$ tinham consumido bebida alcoólica nos últimos 30 dias; $66,1 \%$ eram inativos fisicamente e 55,7\% totalizavam mais de 3 horas diárias de tempo de tela (Tabela 1). A idade e a escolaridade se associaram à HAS em nível bivariado $(\mathrm{p}<0,05)$, enquanto o tabagismo foi selecionado para o ajuste multivariado dos dados $(\mathrm{p}<0,20)$ (Tabela 1).

As informações coletadas indicaram ainda baixo consumo de frutas e de sucos naturais (18\%) e de verduras e legumes (30\%). A maioria dos indivíduos que participou do estudo consumia carne com excesso de gordura, bebia refrigerante mais de 5 vezes na semana e adicionava sal à comida pronta. Contudo, mais da metade consumia feijão regularmente. Em relação aos hábitos alimentares, nenhuma variável associou à HAS em nível bivariado, mas o consumo de verduras e legumes foi selecionado para o ajuste multivariado dos dados $(\mathrm{p}<0,20)$ (Tabela 2$)$. 
Tabela 1

Características demográficas, socioeconômica e de estilo de vida e suas associações com a hipertensão arterial em indígenas Krenak. Resplendor, Minas Gerais, Brasil, 2016.

\begin{tabular}{|c|c|c|c|c|c|}
\hline \multirow[t]{2}{*}{ Variáveis } & \multirow{2}{*}{$\begin{array}{c}\text { População } \\
\text { n (\%) }\end{array}$} & \multicolumn{4}{|c|}{ Hipertensão arterial } \\
\hline & & $\%$ & $\mathbf{R P}$ & IC95\% & Valor de $p$ * \\
\hline Sexo & & & & & 0,322 \\
\hline Feminino & $96(52,5)$ & 27,6 & 1,00 & - & \\
\hline Masculino & $87(47,5)$ & 34,4 & 1,24 & $0,80-1,93$ & \\
\hline Idade (anos) & & & & & $<0,001$ \\
\hline $18-29$ & $62(33,9)$ & 6,4 & 1,00 & - & \\
\hline $30-39$ & $72(39,3)$ & 31,9 & 4,95 & $1,80-13,57$ & \\
\hline $40-49$ & $25(13,7)$ & 52,0 & 8,06 & $2,89-22,41$ & \\
\hline $50-59$ & $12(6,6)$ & 66,8 & 10,33 & $3,68-28,99$ & \\
\hline 60 ou mais & $12(6,6)$ & 75,0 & 11,62 & $4,25-31,76$ & \\
\hline Situação conjugal & & & & & 0,326 \\
\hline Sem cônjuge & $40(21,8)$ & 37,5 & 1,00 & - & \\
\hline Com cônjuge & $143(78,1)$ & 29,4 & 0,78 & $0,48-1,25$ & \\
\hline Escolaridade (anos) & & & & & 0,007 \\
\hline 10 ou mais & $73(39,9)$ & 27,4 & 1,00 & - & \\
\hline $6-9$ & $42(23,0)$ & 16,6 & 0,60 & $0,28-1,31$ & \\
\hline $0-5$ & $68(37,2)$ & 44,1 & 1,61 & $1,01-2,55$ & \\
\hline Tabagismo ** & & & & & 0,068 \\
\hline Não fumante & $128(70,0)$ & 26,6 & 1,00 & - & \\
\hline Ex-fumante & $29(15,9)$ & 48,3 & 1,81 & $1,12-2,92$ & \\
\hline Fumante & $26(14,2)$ & 34,6 & 1,30 & $0,71-2,38$ & \\
\hline Consumo de álcool (vezes/mês) *** & & & & & 0,388 \\
\hline Não & $133(72,7)$ & 29,3 & 1,00 & - & \\
\hline $1-2$ & $24(13,1)$ & 25,0 & 0,85 & $0,41-1,79$ & \\
\hline $3-6$ & $11(6,0)$ & 45,5 & 1,55 & $0,77-3,12$ & \\
\hline$>7$ & $15(8,2)$ & 46,7 & 1,59 & $0,87-2,91$ & \\
\hline Atividade física \# & & & & & 0,220 \\
\hline Ativo & $32(17,5)$ & 18,8 & 1,00 & - & \\
\hline Insuficiente ativo & $30(16,4)$ & 30,0 & 1,60 & $0,65-3,96$ & \\
\hline Inativo & $121(66,1)$ & 34,7 & 1,85 & $0,86-3,97$ & \\
\hline Tempo de tela (horas/dia) & & & & & 0,693 \\
\hline$<3$ & $81(44,3)$ & 29,6 & 1,00 & - & \\
\hline$\geq 3$ & $102(55,7)$ & 32,4 & 1,09 & $0,70-1,69$ & \\
\hline
\end{tabular}

IC95\%: intervalo de 95\% de confiança; RP: razão de prevalência.

Nota: hipertensão arterial (pressão arterial sistólica > 140mmHg e/ou pressão arterial diastólica > 90mmHg e/ou em uso de medicamento anti-hipertensivo).

* Valor de p do teste de qui-quadrado de Pearson;

** Qualquer indivíduo que fuma, independentemente da frequência e intensidade;

*** Consumo pesado episódico de bebida alcóolica nos últimos 30 dias de cinco ou mais doses para homem e quatro ou mais doses para mulher (uma dose = uma lata de cerveja, uma taça de vinho ou uma dose de cachaça, whisky);

\# Inativo (< 1 minuto/semana), insuficiente ativo (> 1 a 149 minutos/semana), ativo (> 150 minutos/semana). 
Tabela 2

Características dos hábitos alimentares e suas associações com a hipertensão arterial em indígenas Krenak. Resplendor, Minas Gerais, Brasil, 2016.

\begin{tabular}{|c|c|c|c|c|c|}
\hline \multirow[t]{2}{*}{ Variáveis } & \multirow{2}{*}{$\begin{array}{c}\text { População } \\
\text { n (\%) }\end{array}$} & \multicolumn{4}{|c|}{ Hipertensão arterial } \\
\hline & & $\%$ & $\mathbf{R P}$ & IC95\% & Valor de $p$ * \\
\hline Adição de sal & & & & & 0,441 \\
\hline Não & $88(48,0)$ & 33,7 & 1,00 & - & \\
\hline Sim & $95(51,9)$ & 28,4 & 0,84 & $0,54-1,30$ & \\
\hline Feijão & & & & & 0,879 \\
\hline Consumo regular ** & $133(72,7)$ & 30,8 & 1,00 & - & \\
\hline Consumo irregular $\star \star \star$ & $50(27,3)$ & 32,0 & 1,03 & $0,64-1,67$ & \\
\hline Frutas e sucos naturais & & & & & 0,259 \\
\hline Consumo regular ** & $33(18,0)$ & 39,4 & 1,00 & - & \\
\hline Consumo irregular $\star \star \star$ & $150(82,0)$ & 29,3 & 0,74 & $0,45-1,21$ & \\
\hline Verduras e legumes & & & & & 0,090 \\
\hline Consumo regular ** & $55(30,0)$ & 40,0 & 1,00 & - & \\
\hline Consumo irregular $\star \star \star$ & $128(70,0)$ & 27,3 & 0,68 & $0,44-1,05$ & \\
\hline Carne com gordura & & & & & 0,394 \\
\hline Retira excesso & $68(37,2)$ & 29,7 & 1,00 & - & \\
\hline Não retira excesso & $115(62,8)$ & 37,1 & 1,24 & $0,75-2,05$ & \\
\hline Refrigerante (vezes/semana) & & & & & 0,883 \\
\hline 5 ou mais & $117(63,9)$ & 30,8 & 1,00 & - & \\
\hline$<5$ & $66(36,1)$ & 31,8 & 1,03 & $0,66-1,61$ & \\
\hline
\end{tabular}

IC95\%: intervalo de 95\% de confiança; RP: razão de prevalência.

Nota: hipertensão arterial (pressão arterial sistólica > 140mmHg e/ou pressão arterial diastólica > 90mmHg e/ou em uso de medicamento anti-hipertensivo).

* Valor de p do teste de qui-quadrado de Pearson;

** Cinco ou mais dias da semana;

*** Quatro ou menos dias da semana.

Aproximadamente três quartos dos participantes apresentaram excesso de peso segundo a classificação do IMC e 57\% apresentaram obesidade abdominal de acordo com a classificação do PC. Em relação à glicemia, 17,5\% dos indivíduos apresentaram hiperglicemia. Todas as variáveis antropométricas e a glicemia capilar foram associadas à HAS em nível bivariado $(\mathrm{p}<0,05)$ (Tabela 3$)$.

A variável IMC não foi inserida na análise multivariada, uma vez que apresentou forte correlação com a variável PC (coeficiente de correlação de Pearson = 0,89). Após o ajuste multivariado dos dados, as variáveis idade, obesidade abdominal e hiperglicemia permaneceram associadas à HAS (Tabela 4).

\section{Discussão}

No presente estudo, verificou-se elevada prevalência de HAS $(31,2 \%)$ associada independentemente à idade, obesidade abdominal e hiperglicemia. Tal achado foi semelhante às descritas em estudos realizados com os Mura, do Amazonas (29\%) 15, entre os Mehináku, Waurá e Yawalaptí, no Alto Xingu $(37,7 \%) 16$ e os Suyá (26,7\%), do Mato Grosso 28. Estudo realizado com dois grupos indígenas que vivem no Espírito Santo, na Região Sudeste do país, a mesma dos Krenak, observou prevalência de HAS (20,8\%) inferior ao da presente investigação 29. Entretanto, é importante ter cautela ao comparar os dados, pois a diversidade étnica dos povos indígenas brasileiros e a localização de suas TI dificultam análises gerais em relação ao perfil de saúde e nutrição ${ }^{5}$. No entanto, pode-se observar que a HAS acomete um número importante de indígenas brasileiros. 
Tabela 3

Características relacionadas às morbidades e suas associações com a hipertensão arterial em indígenas Krenak. Resplendor, Minas Gerais, Brasil, 2016.

\begin{tabular}{|c|c|c|c|c|c|}
\hline \multirow[t]{2}{*}{ Variáveis } & \multirow{2}{*}{$\begin{array}{c}\text { População } \\
\text { n (\%) }\end{array}$} & \multicolumn{4}{|c|}{ Hipertensão arterial } \\
\hline & & $\%$ & $\mathbf{R P}$ & IC95\% & Valor de $p$ * \\
\hline $\mathrm{IMC}\left(\mathrm{kg} / \mathrm{m}^{2}\right)$ & & & & & 0,005 \\
\hline Normal & $47(25,68)$ & 14,9 & 1,00 & - & \\
\hline Excesso de peso & $136(74,32)$ & 36,6 & 2,46 & $1,20-5,07$ & \\
\hline Obesidade abdominal ** & & & & & $<0,001$ \\
\hline Normal & $78(42,62)$ & 11,5 & 1,00 & - & \\
\hline Risco elevado & $43(23,50)$ & 30,2 & 2,62 & $1,21-5,63$ & \\
\hline Risco muito elevado & $62(33,88)$ & 56,4 & 4,89 & $2,54-9,40$ & \\
\hline Hiperglicemia (mg/dL) & & & & & 0,003 \\
\hline Não & $151(82,51)$ & 26,5 & 1,00 & - & \\
\hline Sim & $32(17,49)$ & 53,1 & 2,00 & $1,31-3,05$ & \\
\hline
\end{tabular}

IC95\%: intervalo de 95\% de confiança; IMC: índice de massa corporal; RP: razão de prevalência.

Nota: hipertensão arterial (pressão arterial sistólica $>140 \mathrm{mmHg}$ e/ou pressão arterial diastólica >90mmHg e/ou em uso de medicamento anti-hipertensivo).

* Valor de $p$ do teste de qui-quadrado de Pearson;

** Perímetro da cintura: normal < $80 \mathrm{~cm}$ para mulheres ou $94 \mathrm{~cm}$ para homens; risco elevado $>80 \mathrm{~cm}$ para mulheres ou $>94 \mathrm{~cm}$ para homens; risco muito elevado $>88 \mathrm{~cm}$ para mulheres ou $>102 \mathrm{~cm}$ para homens.

Estudo de revisão sistemática com metanálise incluiu 23 artigos publicados no período de 1970 a 2014 sobre HAS em indígenas brasileiros, e mostrou que a prevalência combinada de hipertensão nesse período foi de 6,2\% (IC95\%: 3,1\%-10,3\%) e odds ratio de 1,2 (IC95\%: 1,07-1,18), ou seja, um aumento de $12 \%$ ao ano nas chances de um indígena apresentar HAS 13. A baixa prevalência apresentada pela metanálise quando comparada aos estudos citados acima pode ser justificada pela ausência de HAS revelada em quase metade dos estudos incluídos na revisão sistemática, que datam desde 1970, quando a prevalência era praticamente inexistente.

As prevalências da HAS encontrada na maioria dos estudos mencionados foram ligeiramente superiores àquelas registradas na população brasileira não indígena. Segundo a Pesquisa Nacional de Saúde 30 , a prevalência da HAS registrada em adultos brasileiros não indígenas foi de 21,4\%. Estudos com populações indígenas de outras partes do mundo também apresentam prevalência da HAS superiores a outros segmentos populacionais do mesmo país ou região ${ }^{31,32}$. Isso reflete diferenças marcantes nos indicadores e no acesso aos serviços básicos de saúde entre indígenas e não indígenas. Além disso, o achado indica o impacto das importantes transformações socioeconômicas e culturais que se manifestam nos estilos de vida e que possuem importantes reflexos na saúde dos povos indígenas brasileiros. Vale ressaltar as prevalências de fatores de risco para DCNT como o tabagismo, o consumo de bebidas alcoólicas, o sedentarismo e o tempo de tela entre os Krenak avaliados neste estudo, superiores àquelas verificadas para a população adulta não indígena brasileira 7 .

Ademais, as populações tradicionais apresentam forte relação de agravos à saúde provocada por situações como marginalização, dominação, opressão e discriminação, o que culmina no aumento da carga de doenças. A importância e o potencial impacto deste tipo de realidade na etiologia das DCNT entre os povos indígenas têm sido reconhecida em outros contextos indígenas no país 17,18, e parece fazer sentido também nos Krenak.

No presente estudo, a prevalência da HAS foi maior nos indígenas do sexo masculino ainda que o resultado não tenha sido estatisticamente significativo. Por outro lado, análises adicionais, usando ponto de corte para pressão arterial compatível com a classificação de pré-hipertensão 8 , o desfecho manteve-se maior para os homens, sendo tal diferença estatisticamente significativa (dados não apresentados; $\mathrm{p}<0,05)$. Resultados semelhantes foram encontrados em um estudo realizado na TI Xapecó 
Tabela 4

Modelo final hierarquizado de associação independente dos fatores associados à hipertensão arterial em indígenas Krenak. Resplendor, Minas Gerais, Brasil, 2016.

\begin{tabular}{|c|c|c|c|c|c|c|c|c|c|}
\hline \multirow[t]{2}{*}{ Variáveis } & \multicolumn{3}{|c|}{ Bloco 1} & \multicolumn{3}{|c|}{ Bloco 1 + Bloco 2} & \multicolumn{3}{|c|}{ Bloco $1+$ Bloco 2 + Bloco 3} \\
\hline & $\mathbf{R P}$ & IC95\% & Valor de p * & $\mathbf{R P}$ & IC95\% & Valor de $p$ * & $\mathbf{R P}$ & IC95\% & Valor de p * \\
\hline \multicolumn{10}{|l|}{ Idade (anos) } \\
\hline $18-29$ & 1,00 & - & - & 1,00 & - & - & 1,00 & - & - \\
\hline $30-39$ & 4,95 & $1,81-13,58$ & 0,002 & 4,53 & $1,64-12,48$ & 0,003 & 3,66 & $1,32-10,15$ & 0,013 \\
\hline $40-49$ & 8,06 & $2,90-22,41$ & $<0,001$ & 7,29 & $2,56-20,71$ & $<0,001$ & 5,30 & $1,85-15,19$ & 0,002 \\
\hline $50-59$ & 10,33 & $3,68-28,99$ & $<0,001$ & 9,70 & $3,20-29,39$ & $<0,001$ & 7,00 & 2,32-21,19 & 0,001 \\
\hline 60 ou mais & 11,63 & $4,25-31,77$ & $<0,001$ & 10,77 & $3,34-34,68$ & $<0,001$ & 7,60 & $2,26-25,60$ & 0,001 \\
\hline \multicolumn{10}{|l|}{ Escolaridade (anos) } \\
\hline 10 ou mais & & & & 1,00 & - & - & 1,00 & - & - \\
\hline $6-9$ & & & & 0,82 & $0,34-1,94$ & 0,648 & 0,78 & $0,36-1,69$ & 0,525 \\
\hline $0-5$ & & & & 1,01 & $0,60-1,69$ & 0,984 & 0,89 & $0,55-1,46$ & 0,657 \\
\hline \multicolumn{10}{|l|}{ Tabagismo ** } \\
\hline Não fumante & & & & 1,00 & - & - & 1,00 & - & - \\
\hline Ex-fumante & & & & 0,99 & $0,58-1,68$ & 0,965 & 1,07 & $0,59-1,94$ & 0,834 \\
\hline Fumante & & & & 1,07 & $0,61-1,86$ & 0,822 & 1,00 & $0,59-1,67$ & 0,987 \\
\hline \multicolumn{10}{|l|}{ Verduras e legumes } \\
\hline 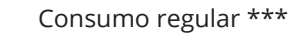 & & & & 1,00 & - & - & 1,00 & - & - \\
\hline Consumo irregular \# & & & & 0,72 & $0,47-1,10$ & 0,125 & 0,70 & $0,47-1,05$ & 0,088 \\
\hline \multicolumn{10}{|l|}{ Obesidade abdominal \#\# } \\
\hline Normal & & & & & & & 1,00 & - & - \\
\hline Risco elevado & & & & & & & 3,06 & $1,29-7,27$ & 0,011 \\
\hline Risco muito elevado & & & & & & & 1,97 & $1,07-3,63$ & 0,029 \\
\hline \multicolumn{10}{|l|}{ Hiperglicemia (mg/dL) } \\
\hline Não & & & & & & & 1,00 & - & - \\
\hline Sim & & & & & & & 1,68 & $1,09-2,57$ & 0,018 \\
\hline
\end{tabular}

IC95\%: intervalo de 95\% de confiança; RP: razão de prevalência.

Nota: hipertensão arterial (pressão arterial sistólica > 140mmHg e/ou pressão arterial diastólica >90mmHg e/ou em uso de medicamento anti-hipertensivo).

* Valor de p da regressão múltipla de Poisson com variâncias robustas;

** Qualquer indivíduo que fuma, independentemente da frequência e intensidade;

*** Cinco ou mais dias da semana;

\# Quatro ou menos dias da semana;

\#\# Perímetro da cintura: normal $<80 \mathrm{~cm}$ para mulheres ou $94 \mathrm{~cm}$ para homens; risco elevado $>80 \mathrm{~cm}$ para mulheres ou $>94 \mathrm{~cm}$ para homens; risco muito elevado $>88 \mathrm{~cm}$ para mulheres ou $>102 \mathrm{~cm}$ para homens.

(Santa Catarina) com a etnia Kaingang ${ }^{14}$. Nesse trabalho, a prevalência da HAS foi de 53,2\% no sexo masculino e 40,7\% no feminino. Um estudo conduzido com os indígenas Aruák (Mato Grosso) também mostrou maiores prevalências da HAS para o sexo masculino (54,7\% para o sexo masculino vs. $21,8 \%$ para o sexo feminino) 16 . Estimativas da OMS apontam maiores prevalências da HAS para o sexo masculino em nível mundial (29,2\% para o sexo masculino $v s .24,8 \%$ para o sexo feminino) 6 . $\mathrm{Na}$ presente pesquisa, a amostra foi formada majoritariamente por indivíduos jovens; apenas 13,2\% das pessoas tinham mais de 50 anos. Isso pode estar relacionado com o fato de a menor prevalência da HAS ter sido encontrada no sexo feminino, uma vez que as mulheres contam com o efeito protetor do hormônio estrogênio até a menopausa, depois disso, elas tendem a apresentar prevalência da HAS semelhante à dos homens 33 .

A situação das mulheres Krenak, com 27,6\% diagnosticadas como hipertensas é mais grave que aquela registrada no INSNPI, tanto em termos nacionais (13,2\%) como nas macrorregiões Sul/ 
Sudeste (17,4\%), onde os Krenak estão localizados. No INSNPI 5, a investigação da HAS foi realizada com mulheres indígenas em idade reprodutiva. É possível, contudo, atribuir parte significativa de tais diferenças às faixas etárias investigadas, já que o INSNPI também incluiu mulheres muito jovens e não avaliaram mulheres acima de 50 anos; nessa faixa etária, a maioria das mulheres Krenak era hipertensa. Se retiradas da amostra, a prevalência da HAS entre as mulheres se aproxima de 20\%, valor próximo dos resultados do INSNPI para a macrorregião.

A relação entre o aumento da idade e a prevalência da HAS foi observada na presente pesquisa, achado semelhante em estudos conduzidos com as etnias Guarani-Mbyá (Rio de Janeiro) 12, Kaingang (Santa Catarina) 14, Suyá (Mato Grosso) 28 e Guarani/Tupinikin (Espírito Santo) 29. Um estudo realizado no final da década de 1980 com a etnia Suruí (Rondônia) apontou associação inversa entre a idade e a HAS. Entretanto, quase 17 anos depois, pesquisadores identificaram correlação positiva e significativa da HAS com aumento da idade 34.

Os fatores relacionados à maior prevalência da HAS com o avançar da idade estão associados às alterações hemodinâmicas, como, por exemplo, a arteriosclerose provocada pela perda da elasticidade, enrijecimento e espessamento das artérias que afeta a homeostase pressórica, e resulta em aumento da resistência periférica e do esforço cardíaco para bombear o sangue 33 .

No presente estudo, dentre as variáveis relacionadas ao hábito alimentar, nenhuma se associou independente à HAS, mas é importante notar que mais da metade dos participantes deste estudo referiu adicionar sal à comida pronta. Um estudo publicado pela Organização Pan-Americana da Saúde (OPAS) 35 estimou que grande parte (70\%) do consumo de sal diário dos brasileiros é proveniente da adição durante a preparação dos alimentos e/ou ao alimento pronto na hora do consumo, valor esse que se assemelha aos indígenas Krenak.

Uma dieta rica em sal aumenta a atividade simpática, provoca a expansão do volume sanguíneo e a estimulação de vários mecanismos compensatórios 33. O estudo internacional INTERSALT 36 realizado no final da década de 1980 avaliou de forma sistematizada a relação entre ingestão de sódio $(\mathrm{Na}+)$ e de potássio $(\mathrm{K}+)$ com a pressão arterial e outras variáveis em 52 populações de 32 países; parte da amostra foi formada por indígenas da etnia Yanomámi (Amazonas). Os resultados desse estudo mostraram que quando comparada ao restante da amostra, seja intrapopulacional ou interpopulacional, os Yanomámi apresentaram as médias mais baixas de pressão arterial, bem como ausência de diagnóstico de HAS e associação positiva entre a ingestão de $\mathrm{Na}+$ e a pressão arterial.

Mancilha-Carvalho \& Silva 37 afirmam que os achados podem ser explicados pelo fato de os Yanomámi apresentarem à época uma dieta pobre em $\mathrm{Na}+$, praticarem atividade física regularmente, terem bom estado nutricional, não ingerirem bebidas alcoólicas, consumirem pouca quantidade de gordura saturada e preservarem seus hábitos culturais, o que reduz o risco de doenças cardiovasculares. É necessário ressaltar ainda que o estudo teve lugar há mais de 30 anos e que os Yanomámi viviam praticamente isolados do contato com não indígenas, o que é pouco comum atualmente. Contudo, não há investigações mais recentes sobre a relação entre o consumo de sal e a pressão arterial em indígenas brasileiros, o que dificulta a extrapolação dos dados encontrados entre os Krenak que possuem hábitos culturais e alimentares diferentes dos Yanomámi. Apesar de não ter encontrado associação, análises adicionais usando ponto de corte para pré-hipertensão 8, a adição de sal se associou ao desfecho (dados não apresentados; $\mathrm{p}<0,05$ ). Portanto, este achado deve ser considerado nas políticas e programas de prevenção e controle da HAS, com incentivo à diminuição do consumo de sal na população indígena.

Estudos realizados a partir da década de 1980 38,39,40 constataram mudanças nos padrões alimentares indígenas em diversos contextos em que as atividades tradicionais de subsistência, como, por exemplo, a caça, a pesca, a coleta e os cultivos nativos com frequência perderam espaço para a aquisição comercial de alimentos e o trabalho remunerado. Este conjunto de mudanças resultou no que alguns autores descrevem como uma dieta progressivamente monótona e com um elevado consumo de alimentos industrializados, além da redução dos níveis de atividade física. Em comunidades aborígenes, as prevalências de DCNT foram avaliadas de acordo com o grau de interação com a cultura alimentar ocidental. A menor prevalência (10\%) de DCNT foi encontrada entre os nativos isolados que tinham sua alimentação baseada em vegetais e frutas, e a maior prevalência (27,9\%) para os nativos aculturados com dieta rica em sódio e calorias 41 . 
No caso do Brasil, a investigação do INSNPI 42 revelou que a maior parte dos domicílios estudados $(96,4 \%)$ compra rotineiramente algum item alimentar, 96,6\% relatou utilização de gordura (óleos, margarinas e banha), 99,6\% de sal e 98,7\% de açúcar. Foi evidenciada também a caça/pesca e coleta para fins alimentares em 64,8\% e 69\%, respectivamente, nos domicílios investigados nas cinco macrorregiões do país. A despeito desse quadro, é importante um olhar cuidadoso, pois muitos vivem em situação de extrema pobreza, dependem de cesta básica e recursos como programas de repasse de verbas para sobreviveram.

Em 2015, as condições de vida dos Krenak foram drasticamente afetadas pelo rompimento da barragem de Fundão que continha rejeitos de mineração de ferro, mantida pela Samarco S/A, uma joint venture entre a companhia Vale do Rio Doce S/A e a empresa australiana BHP Billiton ${ }^{21}$. Toneladas de lama e resíduos químicos chegaram ao Rio Doce, inviabilizando atividades como a pesca, agricultura de subsistência, lazer e prática de rituais sagrados. Os alimentos que antes eram cultivados dentro da própria TI foram substituídos por aqueles comprados em supermercado, em sua maioria industrializados. Como forma de reparar o incidente, a empresa paga indenização mensal e cesta básica para as 126 famílias Krenak que vivem à beira do Rio Doce 21. Apesar de ser um fato recente, e a coleta de dados ocorreu nove meses após o acidente ambiental, pressupõe-se que as mudanças ambientais ocorridas na TI Krenak contribuíram para hábitos de vida sedentários e inserção de alimentos industrializados consumidos de maneira exagerada. Tal situação favorece o risco de desenvolvimento de doenças cardiovasculares, especialmente, a HAS. Alguns estudos 43,44 têm demonstrado que o consumo de dieta rica em gordura e açúcar por um período de 12 a 15 semanas já é capaz de provocar disfunções endoteliais com alteração nos níveis pressóricos. Porém, é importante ressaltar que a TI Krenak fica próxima à zona urbana do Município de Resplendor (34,7km). Assim, mesmo antes do desastre ambiental, a comunidade indígena já tinha acesso a outros tipos de alimentos.

Os resultados da presente pesquisa mostraram ainda alta prevalência de excesso de peso e de obesidade abdominal entre os indígenas Krenak, bem como a associação dessas variáveis à HAS. Além disso, a variável independente obesidade abdominal permaneceu associada à variável dependente HAS no modelo final. Tavares et al. ${ }^{34}$, ao estudarem os níveis pressóricos da etnia Suruí (Rondônia), encontraram associação direta entre os índices antropométricos e verificaram que as médias de PAS e PAD correlacionavam positivamente entre os indivíduos que estavam com sobrepeso, tinham obesidade e/ou valores acima da média normal de PC. Ainda nesse estudo, os autores encontraram associação entre a alteração dos níveis pressóricos e a razão cintura-quadril. No tocante aos indígenas Guaraní-Mbya (Rio de Janeiro), a HAS associou positivamente apenas ao índice antropométrico IMC 12. Coimbra Jr. et al. 45 também constataram resultados semelhantes ao investigarem a etnia Xavánte (Mato Grosso). Similarmente ao resultado obtido no presente estudo, o trabalho de Bressan et al. 14 demonstrou associação positiva da HAS apenas para os indígenas com obesidade abdominal. Meyerfreund et al. 29, ao investigarem a HAS em indígenas das etnias Guarani e Tupinikin (Espírito Santo), encontraram maior prevalência da HAS associada ao IMC e à obesidade abdominal.

Cumpre ressaltar que a obesidade abdominal compreende um importante fator de risco modificável que está associada à HAS e a outras comorbidades, como, por exemplo, a síndrome metabólica e o diabetes 8 . A gordura abdominal, por sua vez, é capaz de interferir nos mecanismos de controle da pressão arterial. Isso ocasiona o aumento da atividade simpática, da resistência insulínica e da reabsorção tubular de sódio e água, e, consequentemente, vasoconstrição e aumento do volume plasmático, o que resulta no aumento da pressão arterial 33.

A glicemia alterada que se manteve associada à HAS no modelo final foi observada em 17,5\% dos indígenas adultos Krenak, e pode estar estreitamente ligada à alta prevalência de excesso de peso nessa população. Estudo com indígenas da Aldeia Jaguapiru (Mato Grosso do Sul) 46, também utilizou a glicemia capilar para determinação dos níveis glicêmicos, e observou prevalência de HAS de 29,7\% e desses, 67,5\% apresentaram hiperglicemia ou tolerância à glicose diminuída. Entre os Xavánte (Mato Grosso), ter hiperglicemia e excesso de peso aumentou o risco para o desenvolvimento de doenças cardiovasculares como a HAS 47.

No que tange às limitações deste trabalho, é possível citar o desenho transversal que mede o evento e o desfecho ao mesmo tempo, não sendo capaz de inferir sobre as mudanças ocorridas ao longo do tempo e garantir a causalidade das associações. A utilização de questões do VIGITEL para 
avaliar hábitos comportamentais neste estudo também é considerada como limitação já que nunca foi utilizado em populações indígenas. Apesar disso, o presente estudo apresenta resultados inéditos em uma amostra representativa de etnia indígena nunca estudada anteriormente em relação à temática da HAS.

Em conclusão, a prevalência da HAS e os fatores associados encontrados nos indígenas Krenak foram semelhantes aos obtidos em outros estudos realizados com povos indígenas brasileiros. Além disso, a prevalência da HAS pode ser entendida como uma consequência das mudanças sociodemográficas, de comportamento e de estilo de vida, bem como das situações de vida adversas impostas a esses povos nas últimas décadas. Tendo em vista esse complexo quadro, estratégias devem ser pensadas para prevenção e controle dos fatores de risco relacionados à DCNT entre os indígenas brasileiros, e que não recaiam apenas em medidas individuais, centradas nos estilos de vida. Na vigência dos fatores de ordem estrutural e de sua magnitude, corre-se o risco de deslocar para a responsabilidade das próprias vítimas e/ou das equipes multidisciplinares de saúde indígena a possibilidade de reversão de um quadro de iniquidade em saúde histórica e socialmente determinada. Por fim, é necessário que o escopo de estudos que lidem com fatores de risco para a HAS seja ampliado, a fim de proporcionar o entendimento acerca das peculiaridades de adoecimento entre as diferentes etnias indígenas do Brasil.

\section{Colaboradores}

C. A. Chagas participou da coleta de informações, análise e interpretação dos dados, elaboração da redação e revisão crítica do conteúdo intelectual. A. M. Pimenta participou da coleta de informações, análise e interpretação dos dados e revisão crítica do conteúdo intelectual. M. A. C. B. M. Viana participou da coleta de informações e revisão crítica do conteúdo intelectual. T. G. Castro, M. S. Leite e M. A. Beinner participaram da elaboração do estudo e revisão crítica do conteúdo intelectual.

\section{Informações adicionais}

ORCID: Cristiane Alvarenga Chagas (0000-00025919-8977); Teresa Gontijo de Castro (0000-00031275-4072); Maurício Soares Leite (0000-00024183-375X); Maria Augusta Corrêa Barroso Magno Viana (0000-0002-1122-1197); Mark Anthony Beinner (0000-0002-0980-8976); Adriano Marçal Pimenta (0000-0001-7049-7575).

\section{Agradecimentos}

Os autores agradecem ao povo Krenak das aldeias estudadas e à equipe de saúde indígena local, pela abertura e receptividade ao estudo, ao Distrito Sanitário Especial Indígena Minas Gerais e Espírito Santo pelas informações providas, ao Conselho Nacional de Desenvolvimento Científico e Tecnológico $(\mathrm{CNPq})$ pelo apoio financeiro (processo no 401777/2015-4).

\section{Referências}

1. Anderson I, Robson B, Connolly M, Al-Yaman $\mathrm{F}$, Bjertness $\mathrm{E}$, King $\mathrm{A}$, et al. Indigenous and tribal peoples' health (The-Lowitja Institute Global Collaboration): a population study. Lancet 2016; 388:131-57.

2. Swinburn B, Kraak V, Allender S, Atkins VJ, Baker P, Bograd JR, et al. The global syndemic of obesity, undernutrition, and climate change: The Lancet Commission report. Lancet 2019; 393:791-846.

3. Mikkelsen C, editor. The indigenous world 2013. Copenhagen: International Work Group For Indigenous Affairs; 2013.

4. Instituto Brasileiro de Geografia e Estatística. Censo Demográfico 2010: características gerais dos indígenas. Resultados do universo. Rio de Janeiro: Instituto Brasileiro de Geografia e Estatística; 2012.

5. Coimbra Jr. CEA, Santos RV, Welch JR, Cardoso AM, de Souza MC, Garnelo L, et al. The First National Survey of Indigenous People's Health and Nutrition in Brazil: rationale, methodology, and overview of results. BMC Public Health 2013; 13:52.

6. World Health Organization. A global brief on hypertension. Geneva: World Health Organization; 2013.

7. Departamento de Análise de Situação de Saúde, Secretaria de Vigilância em Saúde, Ministério da Saúde. Vigilância de fatores de risco e proteção para doenças crônicas por inquérito telefônico: VIGITEL 2016. Brasília: Ministério da Saúde; 2017.

8. Sociedade Brasileira de Cardiologia. 7a Diretriz Brasileira de Hipertensão Arterial. Arq Bras Cardiol 2016; 107(3 Suppl 3):1-82. 
9. Oliver WJ, Cohen EL, Neel JV. Blood pressure, sodium intake, and sodium related hormones in the Yanomamo Indians, a "no-salt" culture. Circulation 1975; 52:146-51.

10. Mancilha-Carvalho JJ, Baruzzi RG, Howard PF, Poulter N, Alpers MP, Franco LJ, et al. Blood pressure in four remote populations in the INTERSALT Study. Hypertension 1989; 14238-46.

11. Nascimento JRL, Miranda RA, Xavier FB, Menezes RC. Hipertensão arterial em índios adultos da tribo Tembé, nordeste do Pará. Rev Para Med 1998; 12:45-8.

12. Cardoso AM, Mattos IE, Koifman RJ. Prevalência de fatores de risco para doenças cardiovasculares na população Guaraní-Mbyá do Estado do Rio de Janeiro. Cad Saúde Pública 2001; 17:345-54.

13. Souza Filho ZA, Ferreira AA, Santos B, Pierin AMG. Prevalência de hipertensão arterial em indígenas do Brasil: uma revisão sistemática com meta-análise. Rev Esc Enferm USP 2015; 49:1016-26.

14. Bressan D, Bastos JL, Leite MS. Epidemiology of high blood pressure among the Kaingang people on the Xapecó Indigenous Land in Santa Catarina State, Brazil, 2013. Cad Saúde Pública 2015; 31:331-44.

15. Ferreira AA, Souza-Filho ZA, Gonçalves MJ, Santos J, Pierin AM. Relationship between alcohol drinking and arterial hypertension in indigenous people of the Mura ethnics, Brazil. PLoS One 2017; 12:e0182352.

16. Gimeno SGA, Rodrigues D, Pagliaro H, Cano EN, Lima EES, Baruzzi RG. Perfil metabólico e antropométrico de índios Aruák: Mehináku, Waurá e Yawalapití, Alto Xingu, Brasil Central, 2000/2002. Cad Saúde Pública 2007; 23:194654.

17. Basta PC, Orellana JD, Arantes R. Perfil epidemiológico dos povos indígenas no Brasil: notas sobre agravos selecionados. In: Garnelo L, Pontes NA, organizadores. Saúde indígena: uma introdução ao tema. Brasília: Secretaria de Educação Continuada, Alfabetização, Diversidade e Inclusão, Ministério da Educação; 2012. p. 156-83.

18. Pagliaro H, Azevedo MM, Santos RV. Demografia dos povos indígenas no Brasil: um panorama crítico. In: Pagliaro H, Azevedo MM, Santos RV, organizadores. Demografia dos povos indígenas no Brasil. Rio de Janeiro: Editora Fiocruz; 2005. p. 11-32.

19. Centro de Documentação Eloy Ferreira da Silva. Povos indígenas em Minas Gerais: quem são? http://www.cedefes.org.br/povos-indige nas-destaque/ (acessado em 01/Dez/2017).

20. Paraíso MHB. Krenak. Instituto Socioambiental/Povos Indígenas no Brasil. https://pib.so cioambiental.org/pt/povo/krenak/253 (acessado em 27/Abr/2019).
21. Clínica de Direitos Humanos da Universidade Federal de Minas Gerais. Maior desastre ambiental do país completa dois anos: direito individual das famílias atingidas pelo rompimento da barragem da Samarco prescreve em um ano e indenizações ainda não foram concluídas. https://ufmg.br/comunicacao/noti cias/maior-desastre-ambiental-do-pais-com pleta-dois-anos (acessado em 02/Nov/2017).

22. World Health Organization. Physical status: the use and interpretation of anthropometry. Geneva: World Health Organization; 1995.

23. Lipschitz DA. Screening for nutritional status in the elderly. Prim Care 1994; 21:55-67.

24. National Heart, Lung, and Blood Institute. Detection, evaluation, and treatment of high blood cholesterol in adults (Adult Treatment Panel III). https://www.nhlbi.nih.gov/files/do cs/resources/heart/atp-3-cholesterol-full-re port.pdf (acessado em 15/Dez/2017).

25. Sociedade Brasileira de Diabetes. Diretrizes da Sociedade Brasileira de Diabetes 2015-2016. São Paulo: Sociedade Brasileira de Diabetes; 2016.

26. Victora CG, Huttly SR, Fuchs SC, Olinto MT. The role of conceptual frameworks in epidemiological analysis: a hierarchical approach. Int J Epidemiol 1997; 26:224-7.

27. Barbosa JMV. Prevalência e fatores associados à pressão arterial elevada no povo indígena Xukuru do Ororubá, Pesqueira-PE, 2010 [Dissertação de Mestrado]. Recife: Centro de Pesquisa Aggeu Magalhães, Fundação Oswaldo Cruz; 2013.

28. Salvo VL, Rodrigues D, Baruzzi RG, Pagliaro H, Gimeno SG. Perfil metabólico e antropométrico dos Suyá. Parque Indígena do Xingu, Brasil Central. Rev Bras Epidemiol 2009; 12:458-68.

29. Meyerfreund D, Gonçalves C, Cunha R, Pereira AC, Krieger JE, Mill JG. Age-dependent increase in blood pressure in two different Native American communities in Brazil. J Hypertens 2009; 27:1753-60.

30. Instituto Brasileiro de Geografia e Estatística. Pesquisa Nacional de Saúde: percepção do estado de saúde, estilo de vida e doenças crônicas. Rio de Janeiro: Instituto Brasileiro de Geografia e Estatística; 2014.

31. Schumacher C, Ferucci ED, Lanier AP, Slattery ML, Schraer CD, Raymer TW, et al. Metabolic syndrome: prevalence among American Indian and Alaska native people living in the Southwestern United States and in Alaska. Metab Syndr Relat Disord 2008; 6:267-73.

32. Romero C, Zavaleta C, Cabrera L, Gilman RH, Miranda JJ. Hipertensión arterial y obesidad en indígenas asháninkas de la región Junín, Perú. Rev Peru Med Exp Salud Pública 2014; 31:78-83. 
33. Reckelhoff JF. Gender differences in the regulation of blood pressure. Hypertension 2001; 37:1199-208.

34. Tavares FG, Coimbra Jr. CE, Cardoso AM. Níveis tensionais de adultos indígenas Suruí, Rondônia, Brasil. Ciênc Saúde Colet 2013; 18:1399-409.

35. Legetic B, Campbell N. Reducing salt intake in the Americas: Pan American Health Organization actions. J Health Commun 2011; 16 Suppl 2:37-48.

36. Intersalt: an international study of electrolyte excretion and blood pressure. Results for 24 hour urinary sodium and potassium excretion. Intersalt Cooperative Research Group. BMJ 1988; 297:319-28.

37. Mancilha-Carvalho JJ, Silva NAS. Os Yanomami no INTERSALT. Arq Bras Cardiol 2003; 80:289-94.

38. Vieira-Filho JPB. Problemas da aculturação alimentar dos Xavante e Bororo. Rev Antropol (São Paulo) 1981; 24:37-40.

39. Coimbra Jr. CEA, Flowers NM, Salzano FM, Santos RV. The Xavánte in transition: health, ecology and bioanthropology in Central Brazil. Ann Arbor: University of Michigan Press; 2002.

40. Leite MS, Santos RV, Coimbra Jr. CEA, Gugelmin SA. Alimentação e nutrição dos povos indígenas no Brasil. In: Kac G, Sichieri R, Gigante DP, organizadores. Epidemiologia nutricional. 9a Ed. Rio de Janeiro: Atheneu; 2009. p. 503-17.

41. Kaholokula JK, Nacapoy AH, Grandinetti A, Chang HK. Association between acculturation modes and type 2 diabetes among Native Hawaiians. Diabetes Care 2008; 31:698-700.
42. Cardoso AM, Horta BL, Coimbra Jr CEA, coordenadores. Inquérito Nacional de Saúde e Nutrição dos Povos Indígenas. Rio de Janeiro: Fundação Nacional de Saúde/ABRASCO/Banco Mundial; 2009.

43. Naderali EK, Brown MJ, Pickavance LC, Wilding JP, Doyle PJ, Williams G. Dietary obesity in the rat induces endothelial dysfunction without causing insulin resistance: a possible role for triacylglycerols. Clin Sci (Lond) 2001; 101:499-506.

44. Naderali EK, Williams G. Prolonged endothelial-dependent and -independent arterial dysfunction induced in the rat by short-term feeding with a high-fat, high-sucrose diet. Atherosclerosis 2003; 166:253-9.

45. Coimbra Jr. CE, Chor D, Santos RV, Salzano FM. Blood pressure levels in Xavánte adults from the Pimentel Barbosa Indian Reservation, Mato Grosso, Brazil. Ethn Dis 2001; 11:232-40.

46. Oliveira GF, Oliveira TRR, Rodrigues FF, Corrêa LF, Ikejiri AT, Casulari LA. Prevalência de diabetes melito e tolerância à glicose diminuída nos indígenas da Aldeia Jaguapiru, Brasil. Rev Panam Salud Pública 2011;29:315-21.

47. Soares LP, Dal Fabbro AL, Silva AS, Sartorelli DS, Franco LF, Kuhn PC, et al. Cardiovascular risk in Xavante indigenous population. Arq Bras Cardiol 2018; 110:542-50. 


\section{Abstract}

This cross-sectional study in 2016 aimed to describe the estimated prevalence of systemic arterial hypertension and associated factors in Krenak adults and elderly in an indigenous community located along the Rio Doce in eastern Minas Gerais state, Brazil. We measured weight, height, waist circumference, systolic blood pressure, diastolic blood pressure, and capillary blood glucose. Sociodemographic and lifestyle information was obtained from a face-to-face questionnaire. Poisson regression models were constructed to estimate independent associations between the target variables and hypertension. Prevalence of hypertension was 31.2\% (95\%CI: 24.4-37.9) in Krenak indigenous. The final model showed an independent association with increasing age, abdominal obesity, and hyperglycemia. The results highlight the need for effective measures in prevention, diagnosis, and follow-up of modifiable risk factors for hypertension, since high prevalence of this condition was observed in the Krenak indigenous community.

Hypertension; South American Indians; Risk Factors; Nutrition Assessment; Community Health Nursing

\section{Resumen}

Estudio transversal realizado en 2016, cuyo objetivo fue describir la prevalencia estimada y los factores asociados a la hipertensión arterial sistémica entre adultos y ancianos Krenak, en Terra Indígena localizada en la ribera del río Doce, en la región este de Minas Gerais, Brasil. Se midieron peso, estatura, perimetro de la cintura, presión arterial sistólica, presión arterial diastólica y glucemia capilar. Informaciones sociodemográficas y de estilo de vida se obtuvieron vía la aplicación de un cuestionario cara-a-cara. Modelos de regresión de Poisson se construyeron para estimar la asociación independiente entre las variables de interés y la hipertensión arterial sistémica. La prevalencia de la hipertensión arterial sistémica fue de $31,2 \%$ (IC95\%: 24,4-37,9) entre los indígenas Krenak. Hubo asociación independiente en el modelo final para el aumento de edad, obesidad abdominal e hiperglicemia. Se destaca la necesidad de acciones eficaces de prevención, de diagnóstico y acompanamiento frente a los factores modificables de la hipertensión arterial sistémica, ya que se observó una elevada prevalencia de esa condición en la comunidad indígena Krenak.

Hipertensión; Indios Sudamericanos; Factores de Riesgo; Evaluación Nutricional; Enfermería en Salud Comunitaria
Recebido em 30/Out/2018

Versão final reapresentada em 24/Mai/2019

Aprovado em 26/Jun/2019 